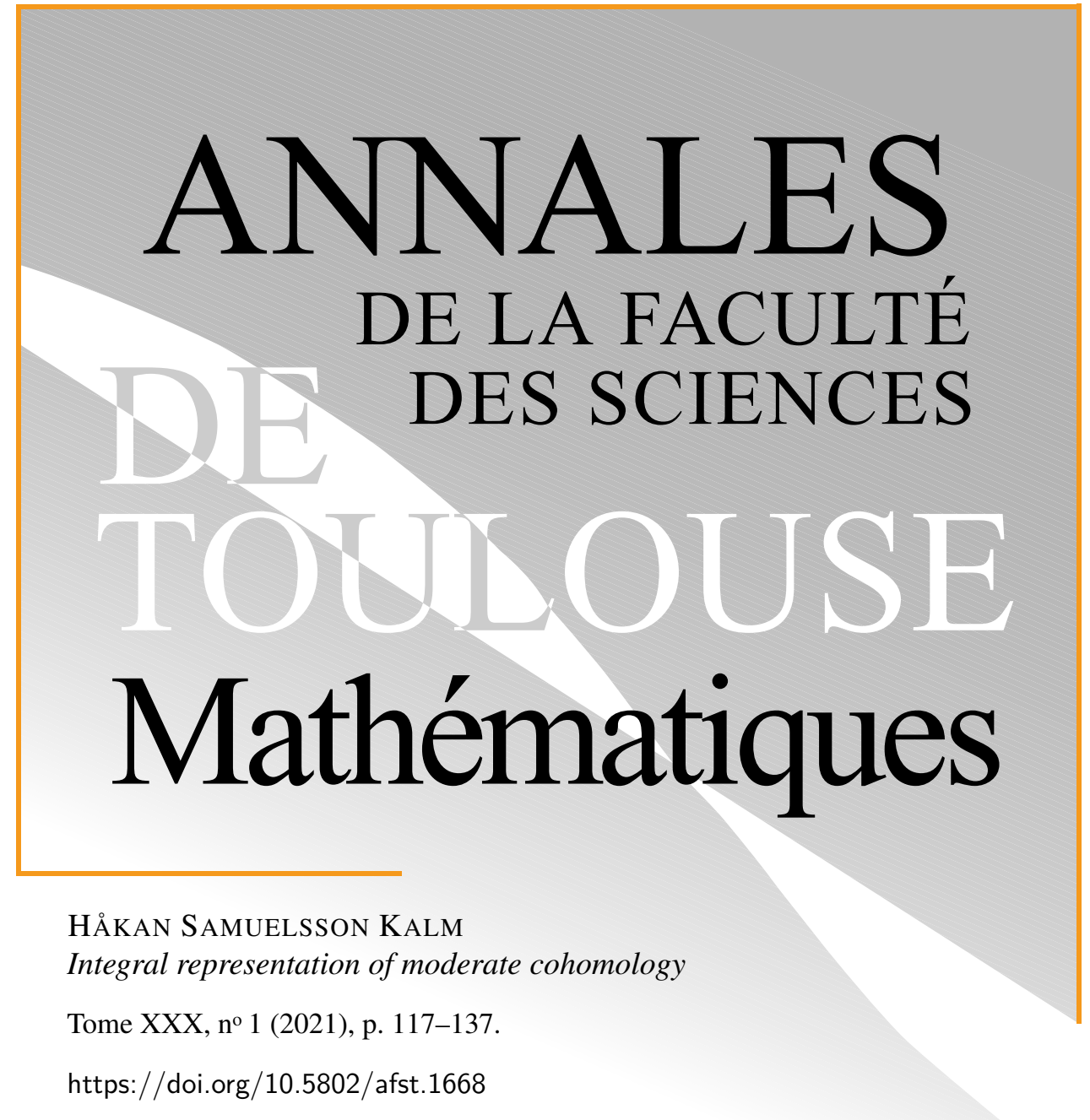

(c) Université Paul Sabatier, Toulouse, 2021.

L'accès aux articles de la revue «Annales de la faculté des sciences de Toulouse Mathématiques » (http://afst.centre-mersenne.org/) implique l'accord avec les conditions générales d'utilisation (http://afst.centre-mersenne.org/legal/). Les articles sont publiés sous la license CC-BY 4.0.

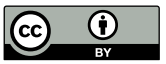

Publication membre du centre

Mersenne pour l'édition scientifique ouverte MERSENNE http://www.centre-mersenne.org/ 


\title{
Integral representation of moderate cohomology ${ }^{(*)}$
}

\author{
HÅKAN SAMUELSSON KALM ${ }^{(1)}$
}

\begin{abstract}
We make the classical Dickenstein-Sessa canonical representation in local moderate cohomology explicit by an integral formula. We also provide a similar representation of the higher local moderate cohomology groups.

RÉSumÉ. - Nous faisons la représentation canonique classique de DickensteinSessa dans la cohomologie modérée locale explicite par une formule intégrale. Nous fournissons également une représentation similaire des groupes de cohomologie modérés locaux supérieurs.
\end{abstract}

\section{Introduction}

Let $M$ be a complex $N$-dimensional manifold, $Y \subset M$ an analytic subset of pure codimension $\kappa$, and $\mu$ a $\bar{\partial}$-closed current on $M$ with support in $Y$. In general there are local obstructions to solving $\bar{\partial} \tau=\mu$ for a current $\tau$ with support in $Y$. For instance, the current of integration, $[Y]$, along $Y$ has locally no $\bar{\partial}$-potential with support in $Y$. Such local obstructions constitute the moderate cohomology sheaf.

The following fundamental result was proved by Dickenstein and Sessa in [17]: If $Y$ is a locally complete intersection and $\mu$ has bidegree $(p, \kappa)$, then there is locally a unique decomposition

$$
\mu=\nu+\bar{\partial} \tau
$$

${ }^{(*)}$ Reçu le 22 mai 2018, accepté le 11 juin 2019.

Keywords: moderate cohomology, integral representation, residue current, coherent ideal sheaf, complex space.

2020 Mathematics Subject Classification: 32A26, 32A27, 32C25, 32C35.

(1) Håkan Samuelsson Kalm, Department of Mathematical Sciences, Division of Algebra and Geometry, University of Gothenburg and Chalmers University of Technology, SE-412 96 Göteborg, Sweden — hasam@chalmers.se

Author partially supported by the Swedish Research Council.

Article proposé par Vincent Guedj. 
where $\nu$ is a "locally residual current" with support in $Y$ and $\tau$ is a current with support in $Y$. Hence, the moderate cohomology sheaf is isomorphic to the sheaf of locally residual currents.

That $\nu$ is a locally residual current here means that $\nu$ locally can be written $\nu=\xi \wedge R^{f}$, where $\xi$ is a holomorphic $p$-form and $R^{f}$ is the ColeffHerrera product, [15],

$$
R^{f}=\bar{\partial}\left(1 / f_{1}\right) \wedge \cdots \wedge \bar{\partial}\left(1 / f_{\kappa}\right)
$$

associated with a tuple $f=\left(f_{1}, \ldots, f_{\kappa}\right)$ of holomorphic functions such that $Y=\left\{f_{1}=\cdots=f_{\kappa}=0\right\}$. The uniqueness of the decomposition (1.1) means that if also $\mu=\nu^{\prime}+\bar{\partial} \tau^{\prime}$ then $\nu=\nu^{\prime}$ and $\bar{\partial} \tau=\bar{\partial} \tau^{\prime}$. The known proofs of the Dickenstein-Sessa decomposition are implicit and so it is a natural problem to find $\nu$ explicitly given $\mu$. The main objective of this paper is to find $\nu$ by an integral formula.

Given $\mu$ the current $\nu$ is unique. However, the factorization $\nu=\xi \wedge R^{f}$ is not. Any $R^{f}$ as above works as long as $g \mu=0$ for all $g$ in the ideal $\left\langle f_{1}, \ldots, f_{\kappa}\right\rangle$. Thus, given $\mu$ and such a choice of $R^{f}$, our problem is to find $\xi$.

From now on $\mathcal{J}$ is a coherent ideal sheaf with zero set $Y$ and $Y$ need not be a locally complete intersection. In this context Björk's formalization of locally residual currents is very useful. Following [14, Section 6.2], a $(p, \kappa)$ current $w$ is a Coleff-Herrera current with support in $Y$ if it is $\bar{\partial}$-closed, satisfies $\overline{\mathcal{J}}_{Y} w=0$, where $\mathcal{J}_{Y}:=\sqrt{\mathcal{J}}$, and has the standard extension property (SEP) with respect to $Y$. That $w$ has the SEP with respect to $Y$ means roughly speaking that it has no mass concentrated on proper analytic subsets of $Y$, see Section 2.1 below for details. The Coleff-Herrera currents with support in $Y$ and the locally residual currents as described above are the same if $Y$ is a locally complete intersection.

Suppose now that $\mathcal{J} \mu=0$; notice that for any given current with support in $Y$ this holds locally for some choice of $\mathcal{J}$ since currents have locally finite order. Then, there is a unique decomposition (1.1), where $\nu$ is a Coleff-Herrera current with support in $Y$ such that $\mathcal{J} \nu=0$ and where $\tau$ can be chosen so that $\mathcal{J} \tau=0$; see, e.g., [6] and [14], cf. also [18]. It is proved in [6] that any such $\nu$ can be written (in a non-unique way) locally as $\xi \cdot R_{\kappa}$. Here, and in what follows, $R_{\kappa}$ is the part of bidegree $(0, \kappa)$ of the Andersson-Wulcan current, [9], associated to a Hermitian locally free resolution of $\mathscr{O}_{M} / \mathcal{J}$ (cf. Section 2.2 below); $R_{\kappa}$ takes values in a vector bundle $E$ and $\xi$ is a holomorphic $p$-form with values in the dual bundle $E^{*}$. If $\mathcal{J}=\left\langle f_{1}, \ldots, f_{\kappa}\right\rangle$ is a locally complete intersection, then the Koszul complex of $f_{1}, \ldots, f_{\kappa}$ is a free resolution of $\mathscr{O}_{M} / \mathcal{J}$ and the associated current then equals $R^{f}$, cf. Example 2.1. 
TheOREM 1.1. - Let $M \subset \mathbb{C}^{N}$ be a pseudoconvex domain and let $M^{\prime} \Subset$ $M$. There is an integral kernel $P(\zeta, z)$ with the following properties:

(i) $\zeta \mapsto P(\zeta, z)$ is a holomorphic p-form in $M^{\prime}$ with values in $E^{*}$;

(ii) $z \mapsto P(\zeta, z)$ is a smooth compactly supported $(N-p, N-\kappa)$-form in $M$;

(iii) if $\mu$ is a $\bar{\partial}$-closed $(p, \kappa)$-current in $M$ with $\mathcal{J} \mu=0$ then, in $M^{\prime}, \nu$ in (1.1) is given by

$$
\nu=\breve{P}_{\mu}:=R_{\kappa} \cdot \int_{z} P(\zeta, z) \wedge \mu(z) .
$$

The integral means the action of $\mu$ on the test form $z \mapsto P(\zeta, z)$. The kernel $P(\zeta, z)$ is explicitly constructed given a free resolution of $\mathscr{O}_{M} / \mathcal{J}$, see Section 3 below.

One application of Theorem 1.1 is to the problem of factorizing cycles. It is shown in [15] that if $\mathcal{J}=\left\langle f_{1}, \ldots, f_{\kappa}\right\rangle$ is a complete intersection and $Z$ is the corresponding fundamental cycle, i.e., $Z=\sum_{j} m_{j} Y_{j}$ where $m_{j}$ are certain multiplicities and $Y_{j}$ are the irreducible components of $Y$, then $[Z]=R^{f} \wedge$ $\mathrm{d} f_{\kappa} \wedge \cdots \wedge \mathrm{d} f_{1} /(2 \pi i)^{\kappa}$. This was globalized to locally complete intersections by Demailly and Passare, [16], and further generalized by Andersson in [3]. Recently, Lärkäng and Wulcan, [22], proved a formula similar to $[Z]=R^{f} \wedge$ $\mathrm{d} f_{\kappa} \wedge \cdots \wedge \mathrm{d} f_{1} /(2 \pi i)^{\kappa}$ for the fundamental cycle of a quite general complex subspace. If $Z$ is any cycle with $|Z|=Y$ we obtain

$$
[Z]=R_{\kappa} \cdot \int_{z} P(\zeta, z) \wedge[Z] .
$$

Indeed, by the decomposition (1.1) and Theorem 1.1, (1.2) holds modulo a term $\bar{\partial} \tau$. However, $[Z]$ already is a Coleff-Herrera current with support in $Y$ so the uniqueness of the decomposition (1.1) implies that $\bar{\partial} \tau=0$.

The Dickenstein-Sessa result gives a canonical representation of the moderate cohomology sheaf associated with $Y$ in degree $\kappa$, which is the lowest degree with non-vanishing cohomology. In Section 4, Theorems 4.2 and 4.3, we give a representation of the moderate cohomology sheaf in degree $q$ with $q>\kappa$.

\section{Acknowledgments}

I would like to thank Mats Andersson and Elizabeth Wulcan for stimulating discussions on the topic of this paper. 


\section{Pseudomeromorphic currents and weighted integral formulas}

\subsection{Pseudomeromorphic currents}

In one complex variable $z$ it is elementary to see that the principal value current $1 / z^{m}$ exists and can be defined, e.g., as the limit as $\epsilon \rightarrow 0$ in the sense of currents of $\chi\left(|h(z)|^{2} / \epsilon\right) / z^{m}$, where $h$ is a non-trivial holomorphic function (or tuple) vanishing at $z=0$ and $\chi$ is a smooth regularization of the characteristic function of $[1, \infty) \subset \mathbb{R}$; for the rest of the paper $\chi$ will denote such a function. The current $1 / z^{m}$ can also be defined as the value at $\lambda=0$ of the analytic continuation of the current-valued function $\lambda \mapsto|h(z)|^{2 \lambda} / z^{m}$. It follows that the residue current $\bar{\partial}\left(1 / z^{m}\right)$ can be computed as the limit of $\bar{\partial} \chi\left(|h(z)|^{2} / \epsilon\right) / z^{m}$ or as the value at $\lambda=0$ of $\lambda \mapsto \bar{\partial}|h(z)|^{2 \lambda} / z^{m}$. Since tensor products of currents are well-defined we can form the current

$$
\tau=\bar{\partial} \frac{1}{z_{1}^{m_{1}}} \wedge \cdots \wedge \bar{\partial} \frac{1}{z_{r}^{m_{r}}} \wedge \frac{\gamma(z)}{z_{r+1}^{m_{r+1}} \cdots z_{M}^{m_{d}}}
$$

in $\mathbb{C}^{d}$, where $m_{1}, \ldots, m_{r}$ are positive integers, $m_{r+1}, \ldots, m_{d}$ are nonnegative integers, and $\gamma$ is a smooth compactly supported form. Notice that $\tau$ is anticommuting in the residue factors $\bar{\partial}\left(1 / z_{j}^{m_{j}}\right)$ and commuting in the principal value factors $1 / z_{k}^{m_{k}}$. We say that a current of the form (2.1) is an elementary pseudomeromorphic current. A current $\mu$ on a complex manifold $M$ is pseudomeromorphic if and only if $\mu$ is a locally finite sum of currents of the form $\pi_{*} \tau$, where $\tau$ is of the form (2.1) and $\pi$ is a holomorphic map from a neighborhood of supp $\gamma$ to $M$, see [11, Theorem 2.15]. Currents on reduced complex spaces are also defined, see [19]. A current $\mu$ on a reduced puredimensional complex space $X$ is pseudomeromorphic if and only if there is a modification $\pi: X^{\prime} \rightarrow X$ with $X^{\prime}$ smooth and a pseudomeromorphic current $\mu^{\prime}$ on $X^{\prime}$ such that $\mu=\pi_{*} \mu^{\prime},[11$, Theorem 2.15]. This yields the subsheaf $\mathscr{P} \mathscr{M}_{X}$ of the sheaf of germs of currents on any reduced pure-dimensional complex space $X$. Notice that, since $\bar{\partial}$ maps an elementary pseudomeromorphic current to a sum of such currents, $\bar{\partial}$ maps $\mathscr{P} \mathscr{M}$ to itself. Moreover, if $X$ and $Z$ are reduced pure-dimensional complex spaces and $\mu \in \mathscr{P} \mathscr{M}(X)$, then $\mu \otimes 1 \in \mathscr{P} \mathscr{M}(X \times Z)$, see [8, Section 2]. Below, we will omit " $\otimes 1$ " and write, e.g., $\mu(x)$ to denote on what variables a current depends.

Dimension PRinciple ([10, Corollary 2.4], [8, Proposition 2.3]). — Let $X$ be a reduced pure-dimensional complex space, let $\mu \in \mathscr{P} \mathscr{M}(X)$, and assume that $\mu$ has support contained in a subvariety $V \subset X$. If $h \in \mathscr{O}_{X}$ vanishes on $V$ then $\bar{h} \mu=\mathrm{d} \bar{h} \wedge \mu=0$. Moreover, if $\mu$ has bidegree $(*, q)$ and $\operatorname{codim}_{X} V>q$, then $\mu=0$. 
Pseudomeromorphic currents can be "restricted" to analytic (or constructible) subsets: Let $\mu \in \mathscr{P} \mathscr{M}(X)$, let $V \subset X$ be an analytic subset, and set $V^{c}:=X \backslash V$. Then the restriction of $\mu$ to the open subset $V^{c}$ has a natural pseudomeromorphic extension $\mathbf{1}_{V^{c}} \mu$ to $X$. In [10], $\mathbf{1}_{V^{c}} \boldsymbol{\mu}$ is defined as the value at 0 of the analytic continuation of the current-valued function $\lambda \mapsto|h|^{2 \lambda} \mu$, where $h$ is any holomorphic tuple with zero set $V ; \mathbf{1}_{V^{c}} \mu$ can also be defined as $\lim _{\epsilon \rightarrow 0} \chi\left(|h|^{2} v / \epsilon\right) \mu$, where $v$ is any smooth strictly positive function, see [11, Lemma 2.6], cf. also [21, Lemma 6]. The current $\mathbf{1}_{V} \mu:=\mu-\mathbf{1}_{V^{c}} \mu$ thus is a pseudomeromorphic current with support contained in $V$, and if supp $\mu \subset V$, then $\mathbf{1}_{V} \mu=\mu$. Moreover, see [11, Section 2.2], if $V$ and $W$ are any constructible subsets then $\mathbf{1}_{V} \mathbf{1}_{W} \mu=\mathbf{1}_{V \cap W} \mu$. A current $\mu \in \mathscr{P} \mathscr{M}(X)$ has the standard extension property (SEP) with respect to an analytic subsets $V \subset X$ if $\mathbf{1}_{W} \mu=0$ for all germs of analytic subsets $W \subset X$ such that $\operatorname{codim}_{V} W \cap V>0$.

Recall that a current on $X$ is said to be semi-meromorphic if it is a principal value current of the form $\alpha / f$, where $\alpha$ is a smooth form and $f$ is a holomorphic function or section of a line bundle such that $f$ does not vanish identically on any component of $X$. Following [8], see also [11, Section 4], we say that a current $a$ on $X$ is almost semi-meromorphic if there is a modification $\pi: X^{\prime} \rightarrow X$ and a semi-meromorphic current $\alpha / f$ on $X^{\prime}$ such that $a=\pi_{*}(\alpha / f)$; if $f$ takes values in $L \rightarrow X^{\prime}$ we need also $\alpha$ to take values in $L \rightarrow X^{\prime}$. If $a$ is almost semi-meromorphic on $X$, then the smallest Zariski-closed set outside of which $a$ is smooth has positive codimension and is denoted $Z S S(a)$, the Zariski-singular support of $a$, see [11, Section 4].

For proofs of the statements in this paragraph we refer to [11, Section 4], see also [8, Section 2]. Let $a$ be an almost semi-meromorphic current on $X$ and let $\mu \in \mathscr{P} \mathscr{M}(X)$. Then there is a unique pseudomeromorphic current $T$ on $X$ coinciding with $a \wedge \mu$ outside of $Z S S(a)$ and such that $\mathbf{1}_{Z S S(a)} T=0$. If $h$ is a holomorphic tuple, or section of a Hermitian vector bundle, such that $\{h=0\}=Z S S(a)$, then $T=\lim _{\epsilon \rightarrow 0} \chi\left(|h|^{2} / \epsilon\right) a \wedge \mu$; henceforth we will write $a \wedge \mu$ in place of $T$. One defines $\bar{\partial} a \wedge \mu$ so that Leibniz' rule holds, i.e., $\bar{\partial} a \wedge \mu:=\bar{\partial}(a \wedge \mu)-(-1)^{\operatorname{deg} a} a \wedge \bar{\partial} \mu$. If $\mu$ is almost semi-meromorphic then $a \wedge \mu$ is almost semi-meromorphic and, in fact, $a \wedge \mu=(-1)^{\operatorname{deg} a \operatorname{deg} \mu} \mu \wedge a$.

The sheaf $\mathscr{C} \mathscr{H}_{Y}^{p}$ of Coleff-Herrera currents on a complex manifold with support in an analytic subset $Y$ of pure codimension $\kappa$ was introduced by Björk in the way described in the introduction. It is proved in [5] that $\mathscr{C H}_{Y}^{p}$ equals the subsheaf of $\mathscr{P} \mathscr{M}^{p, \kappa}$ of germs of $\bar{\partial}$-closed currents with support in $Y$. 


\subsection{Currents associated to generically exact complexes}

Let $M, Y$, and $\mathcal{J}$ be as in the introduction. Suppose that we have a complex

$$
0 \longrightarrow E_{m} \stackrel{f_{m}}{\longrightarrow} \cdots \stackrel{f_{1}}{\longrightarrow} E_{0} \longrightarrow 0
$$

of holomorphic Hermitian vector bundles, with $E_{0}$ being the trivial line bundle, such that the associated sheaf complex $\left(\mathscr{O}\left(E_{\bullet}\right), f_{\bullet}\right)$ is a resolution of $\mathscr{O}_{M} / \mathcal{J}$. The bundle $E:=\bigoplus_{j} E_{j}$ gets a natural superstructure by setting $E^{+}:=\bigoplus_{j} E_{2 j}$ and $E^{-}:=\bigoplus_{j} E_{2 j+1}$. Following [9] we define pseudomeromorphic currents $U$ and $R$ with values in $\operatorname{End}(E)$ associated with the Hermitian complex $\left(E_{\bullet}, f_{\bullet}\right)$. Notice that $\operatorname{End}(E)$ gets an induced superstructure and so spaces of forms and currents with values in $E$ or $\operatorname{End}(E)$ get superstructures as well. Let $f:=\bigoplus_{j} f_{j}$ and set $\nabla:=f-\bar{\partial}$, which then becomes an odd mapping on spaces of forms or currents with values in $E$ such that $\nabla^{2}=0$; notice that $\nabla$ induces an odd mapping $\nabla_{\text {End }}$ on $\operatorname{End}(E)$-valued forms or currents such that $\nabla_{\text {End }}^{2}=0$. Outside of $Y,\left(E_{\bullet}, f_{\bullet}\right)$ is pointwise exact and we let $\sigma_{k}: E_{k-1} \rightarrow E_{k}$ be the pointwise minimal inverse of $f_{k}$. Set $\sigma:=\sigma_{1}+\sigma_{2}+\cdots$ and let $u:=\sigma+\sigma \bar{\partial} \sigma+\sigma(\bar{\partial} \sigma)^{2}+\cdots$. Notice that $u=\sum_{0 \leqslant \ell<k} u_{k}^{\ell}$, where $u_{k}^{\ell}:=\sigma_{k} \bar{\partial} \sigma_{k-1} \cdots \bar{\partial} \sigma_{\ell+1}$ is a $\operatorname{smooth} \operatorname{Hom}\left(E_{\ell}, E_{k}\right)$ valued $(0, k-\ell-1)$-form outside of $Y$. One can show that $\nabla_{\text {End }} u=\operatorname{Id}_{E}$. The form $u$ can be exended as a current across $Y$ by setting

$$
U:=\lim _{\epsilon \rightarrow 0} \chi\left(|F|^{2} / \epsilon\right) u
$$

where $F$ is a (non-trivial) holomorphic tuple vanishing on $Y$, see, e.g., [9, Section 2]. As with $u$ we will write $U=\sum_{0 \leqslant \ell<k} U_{\ell}^{k}$, where now $U_{k}^{\ell}$ is a $\operatorname{Hom}\left(E_{\ell}, E_{k}\right)$-valued $(0, k-\ell-1)$-current; in fact, $U$ is an almost semimeromorphic current, cf., e.g., [11]. The current $R$ is defined by $\nabla_{\text {End }} U=$ $\operatorname{Id}_{E}-R$ and hence $R$ is supported on $Y$ and $f R-\bar{\partial} R=\nabla_{\text {End }} R=0$. Notice that $R$ is an almost semi-meromorphic current plus $\bar{\partial}$ of such a current. One can check that

$$
R=\lim _{\epsilon \rightarrow 0}\left(1-\chi\left(|F|^{2} / \epsilon\right)\right) \operatorname{Id}_{E}+\bar{\partial} \chi\left(|F|^{2} / \epsilon\right) \wedge u .
$$

We write $R=\sum_{0 \leqslant \ell<k} R_{k}^{\ell}$, where $R_{k}^{\ell}$ is a $\operatorname{Hom}\left(E_{\ell}, E_{k}\right)$-valued $(0, k-\ell)$ current. Since $E_{0}$ is the trivial line bundle we have $\operatorname{Hom}\left(E_{0}, E_{k}\right) \simeq E_{k}$ and we may identify $R_{k}^{0}$ with an $E_{k}$-valued current; sometimes we just write $R_{k}$ for $R_{k}^{0}$.

Recall that $\kappa=\operatorname{codim} Y$. It is proved in [9] that $R=R_{\kappa}^{0}+R_{\kappa+1}^{0}+\cdots$ and that a holomorphic function $g$ is in $\mathcal{J}$ if and only if the $E$-valued current $R g$ vanishes. 
Example 2.1. - Suppose that $\mathcal{J}=\left\langle f_{1}, \cdots, f_{\kappa}\right\rangle$ is a complete intersection. Let $E_{1}$ be the trivial bundle of rank $\kappa$ with trivial metric and let $e_{1}, \ldots, e_{\kappa}$ be an orthonormal holomorphic frame. Set $E_{k}=\Lambda^{k} E_{1}$ and let the map $E_{k} \rightarrow E_{k-1}$ be interior multiplication with $\sum_{j} f_{j} e_{j}^{*}$. We get the Koszul complex of $f_{1}, \ldots, f_{\kappa}$; this is exact since $\mathcal{J}$ is a complete intersection. The map $\sigma$ is exterior multiplication by $\sum_{j} \bar{f}_{j} e_{j} /|f|^{2}$. The resulting current $R$ is the Coleff-Herrera product $R^{f}$ times $e_{1} \wedge \cdots \wedge e_{\kappa}$, see [2].

\subsection{Weighted integral formulas}

We apply Andersson's method, [1], of generating weighted integral formulas in a domain $D \subset \mathbb{C}^{N}$. To begin with, suppose that $k(\zeta, z)$ is an integrable $(N, N-1)$-form in $D \times D$ and $p(\zeta, z)$ is a smooth $(N, N)$-form in $D \times D$ such that

$$
\bar{\partial} k(\zeta, z)=\left[\Delta^{D}\right]-p(\zeta, z),
$$

where $\left[\Delta^{D}\right]$ is the current of integration along the diagonal. Applying (2.4) to test forms of the form $\psi_{\epsilon}(z) \wedge \varphi(\zeta)$, where $\psi_{\epsilon}$ is an approximate identity and $\varphi$ is a test form in $D$, one obtains the integral formula

$$
\varphi(z)=\bar{\partial}_{z} \int_{D_{\zeta}} k(\zeta, z) \wedge \varphi(\zeta)+\int_{D_{\zeta}} k(\zeta, z) \wedge \bar{\partial} \varphi(\zeta)+\int_{D_{\zeta}} p(\zeta, z) \wedge \varphi(\zeta)
$$

for all $z \in D$ by letting $\epsilon \rightarrow 0$.

Following [1], to find such $k$ and $p$ let $\eta=\left(\eta_{1}, \ldots, \eta_{N}\right)$ be a holomorphic tuple in $D \times D$ that defines the diagonal and let $\Lambda_{\eta}$ be the exterior algebra spanned by $\Lambda^{0,1} T^{*}(D \times D)$ and the $(1,0)$-forms $\mathrm{d} \eta_{1}, \ldots, \mathrm{d} \eta_{N}$. On forms with values in $\Lambda_{\eta}$ interior multiplication with $2 \pi i \sum \eta_{j} \partial / \partial \eta_{j}$, denoted $\delta_{\eta}$, is defined; set $\nabla_{\eta}=\delta_{\eta}-\bar{\partial}$. Let $s$ be a smooth $(1,0)$-form in $\Lambda_{\eta}$ such that $|s| \lesssim|\eta|$ and $|\eta|^{2} \lesssim\left|\delta_{\eta} s\right|$ and let $B=\sum_{k=1}^{N} s \wedge(\bar{\partial} s)^{k-1} /\left(\delta_{\eta} s\right)^{k}$. It is proved in [1] that then $\nabla_{\eta} B=1-\left[\Delta^{D}\right]$. Identifying terms of top degree we see that $\bar{\partial} B_{N, N-1}=\left[\Delta^{D}\right]$ and so $(2.4)$ is satisfied with $k(\zeta, z)=B_{N, N-1}$ and $p(\zeta, z)=0$. For instance, if we take $s=\partial|\zeta-z|^{2}$ and $\eta=\zeta-z$, then the resulting $B$ is sometimes called the full Bochner-Martinelli form and the term of top degree is the classical Bochner-Martinelli kernel.

Let $D_{1}, D_{2} \subset D$. A smooth section $g(\zeta, z)=g_{0}+\cdots+g_{N}$ of $\Lambda_{\eta}$ over $D_{1} \times D_{2}$ such that $\nabla_{\eta} g=0$ in $D_{1} \times D_{2}$ and $g_{0}(z, z)=1$ for $z \in D^{\prime}:=D_{1} \cap D_{2}$ is called a weight with respect to $D_{1} \times D_{2} ; g_{j}$ is the sum of the terms of $g$ of bidegree $(j, j)$. Notice that the exterior product of two weights again is a weight (with respect to a suitable set). If $g$ is a weight with respect to 
$D_{1} \times D_{2}$, then it follows that $\nabla_{\eta}(g \wedge B)=g-g \wedge\left[\Delta^{D}\right]$ in $D_{1} \times D_{2}$ and, identifying terms of bidegree $(N, N-1)$, we get that

$$
\bar{\partial}(g \wedge B)_{N, N-1}=\left[\Delta^{D}\right]-g_{N}
$$

in $D_{1} \times D_{2}$. It follows that if $\varphi$ is smooth with compact support in $D_{1}$, then $(2.5)$, with $k(\zeta, z)=(g(\zeta, z) \wedge B)_{N, N-1}$ and $p(\zeta, z)=g_{N}(\zeta, z)$, holds in $D_{2}$, and vice versa.

Example 2.2. - Let $D \Subset \mathbb{C}^{N}$ be pseudoconvex and let $K \subset D$ be a holomorphically convex compact subset. Let $\rho$ be a smooth compactly supported function in $D$ that is 1 in a neighborhood of $K$. One can find a smooth form $\widetilde{s}(\zeta, z)=\widetilde{s}_{1}(\zeta, z) \mathrm{d} \eta_{1}+\cdots+\widetilde{s}_{N}(\zeta, z) \mathrm{d} \eta_{N}$, defined for $z$ in a neighborhood of supp $\bar{\partial} \rho$ and $\zeta$ in a neighborhood $\widetilde{D}$ of $K$, such that $\zeta \mapsto \widetilde{s}(\zeta, z)$ is holomorphic and $\delta_{\eta} \widetilde{s}=1$. Then

$$
g(\zeta, z):=\rho(z)-\bar{\partial} \rho(z) \wedge \sum_{k=1}^{N} \widetilde{s} \wedge(\bar{\partial} \widetilde{s})^{k-1},
$$

is a weight with respect to $\widetilde{D} \times D$ that depends holomorphically on $\zeta$ and has compact support in $D_{z}$.

If $D$ is a ball centered at 0 and $\eta=\zeta-z$ one can take $\widetilde{s}=(2 \pi i)^{-1} \bar{z}$. $\mathrm{d} \eta /\left(\zeta \cdot \bar{z}-|z|^{2}\right)$.

Example 2.3. - Let $D \Subset \mathbb{C}^{N}$ be pseudoconvex and assume that there is a free resolution $\left(\mathscr{O}\left(E_{\bullet}\right), f_{\bullet}\right)$ of $\mathscr{O}_{D} / \mathcal{J}$ in $D$. Let $U=U(\zeta)$ and $R=R(\zeta)$ be associated currents, let $U^{\epsilon}$ and $R^{\epsilon}$ be the regularizations given by $(2.2)$ and $(2.3)$, respectively, and let $U_{k}^{\epsilon, \ell}$ and $R_{k}^{\epsilon, \ell}$ be the parts taking values in $\operatorname{Hom}\left(E_{\ell}, E_{k}\right)$. By [4, Proposition 5.3] we can, for $\ell \leqslant k$, find Hefer morphisms $H_{k}^{\ell}$, which are holomorphic sections of $\Lambda_{\eta} \otimes \operatorname{Hom}\left(E^{\zeta}, E^{z}\right)$, such that $H_{k}^{\ell}$ is a holomorphic $k$ - $\ell$-form with values in $\operatorname{Hom}\left(E_{k}^{\zeta}, E_{\ell}^{z}\right)$ and

$$
H_{k}^{k} \uparrow_{\Delta_{D}}=\operatorname{Id}_{E_{k}} \quad \text { and } \quad \delta_{\eta} H_{k}^{\ell}=H_{k-1}^{\ell} f_{k}-f_{\ell+1}(z) H_{k}^{\ell+1}, k>\ell,
$$

where $f_{k}=f_{k}(\zeta)$. One can check that then

$$
G^{\epsilon}:=\sum_{k \geqslant 0} H_{k}^{0} R_{k}^{\epsilon, 0}+f_{1}(z) \sum_{k \geqslant 1} H_{k}^{1} U_{k}^{\epsilon, 0},
$$

is a weight with respect to $D \times D$ for all $\epsilon>0$; cf. [4], [8], and [9].

In the case of the Koszul complex, see Example 2.1, the Hefer morphisms can be chosen as follows. We use the notation of Example 2.1. Let $h_{j}=$ $h_{j}(\zeta, z)$ be holomorphic (1,0)-forms such that $\delta_{\eta} h_{j}=f_{j}(\zeta)-f_{j}(z)$ and set $h=\sum_{j} h_{j} \wedge e_{j}^{*}$. Then interior multiplication by $h^{\wedge k-\ell} /(k-\ell)$ ! define Hefer morphisms $H_{k}^{\ell}$. 


\section{Integral operators associated to an ideal sheaf}

Let $D \Subset \mathbb{C}^{N}$ be pseudoconvex, let $\mathcal{J} \subset \mathscr{O}_{D}$ be a coherent sheaf of ideals in $D$ with $Y=Z(\mathcal{J})$ of pure codimension $\kappa$, and let $D^{\prime} \Subset D$. Let $g(\zeta, z)$ be any weight with respect to $D^{\prime} \times D$ such that $z \mapsto g(\zeta, z)$ has compact support in some $D^{\prime \prime} \Subset D$ for all $\zeta$. In $D^{\prime \prime}$ there is a free resolution $\left(\mathscr{O}\left(E_{\bullet}\right), f_{\bullet}\right)$ of $\mathscr{O}_{D} / \mathcal{J}$, associated currents $U=U(\zeta)$ and $R=R(\zeta)$, and, moreover, in $D^{\prime \prime} \times D^{\prime \prime}$ we can find associated Hefer morphisms $H_{k}^{\ell}$. Then $G^{\epsilon}=H R^{\epsilon}+f_{1}(z) H U^{\epsilon}$ of Example 2.3 is a weight with respect to $D^{\prime \prime} \times D^{\prime \prime}$. It follows that $G^{\epsilon} \wedge g$ is a weight with respect to $D^{\prime} \times D$ and has compact support in $D_{z}$.

Notice, in view of Example 2.2, that we may choose the weight $g$ so that it contains no $d \bar{\zeta}$-differentials and $\zeta \mapsto g(\zeta, z)$ is holomorphic; we then say that $g$ is holomorphic in $\zeta$.

Lemma 3.1. - If $\varphi$ is a test form in $D^{\prime}$, then for all $\epsilon>0$ and all $z \in D$

$$
\begin{aligned}
\varphi(z)=\bar{\partial}_{z} \int_{\zeta}\left(H R^{\epsilon} \wedge g \wedge B\right)_{N, N-1} \wedge \varphi(\zeta) & +\int_{\zeta}\left(H R^{\epsilon} \wedge g \wedge B\right)_{N, N-1} \wedge \bar{\partial} \varphi(\zeta) \\
& +\int_{\zeta}\left(H R^{\epsilon} \wedge g\right)_{N, N} \wedge \varphi(\zeta)+\phi \psi,
\end{aligned}
$$

where $\phi$ is a section of $\mathcal{J}$ and $\psi$ is some test form in $D$. The integrals on the right-hand side are test forms in $D$.

Proof. - Since $G^{\epsilon} \wedge g=H R^{\epsilon} \wedge g+f_{1}(z) H U^{\epsilon} \wedge g$ is a weight with respect to $D^{\prime} \times D$ and the entries of $f_{1}$ are sections of $\mathcal{J}$, it follows from Section 2.3 that the claimed equality holds in $D$. Since $z \mapsto g(\zeta, z)$ has compact support in $D, \psi$ as well as the integrals on the right-hand side are test forms in $D$.

Let $\mu$ be an arbitrary current in $D$. Since $z$ and $\zeta-z$ are independent variables in $D \times D$, the tensor product $B \wedge \mu(z)$ is well-defined, cf. [20, Theorem 5.1.1]. Let $\pi: D_{\zeta} \times D_{z} \rightarrow D_{\zeta}$ be the natural projection. Then, since $z \mapsto g(\zeta, z)$ has compact support,

$$
\pi_{*}\left(H R^{\epsilon} \wedge g \wedge B\right)_{N, N-1} \wedge \mu(z) \quad \text { and } \quad \pi_{*}\left(H R^{\epsilon} \wedge g\right)_{N, N} \wedge \mu(z)
$$

are well-defined current in $D_{\zeta}^{\prime}$. For notational convenience we will often write $\int_{z} \tau$ instead of $\pi_{*} \tau$ for a current $\tau$ in $D \times D$.

Lemma 3.2. - Let $\mu$ be a current in $D$ such that $\mathcal{J} \mu=0$. Then, for all $\epsilon>0$

$$
\begin{aligned}
\mu(\zeta)=\int_{z}\left(H R^{\epsilon} \wedge g \wedge B\right)_{N, N-1} \wedge \bar{\partial} \mu(z)+ & \bar{\partial}_{\zeta} \int_{z}\left(H R^{\epsilon} \wedge g \wedge B\right)_{N, N-1} \wedge \mu(z) \\
& +\int_{z}\left(H R^{\epsilon} \wedge g\right)_{N, N} \wedge \mu(z)
\end{aligned}
$$

holds in $D^{\prime}$. 
Proof. - Let $\varphi$ be a test form in $D^{\prime}$. The action of the current $\int_{z}\left(H R^{\epsilon} \wedge\right.$ $g \wedge B)_{N, N-1} \wedge \bar{\partial} \mu(z)$ on $\varphi(\zeta)$ is by definition the action of $\left(H R^{\epsilon} \wedge g \wedge B\right)_{N, N-1} \wedge$ $\bar{\partial} \mu(z)$ on $\varphi(\zeta) \otimes 1$ and this equals, by [20, Theorem 5.1.1], the action of $\bar{\partial} \mu(z)$ on $\int_{\zeta}\left(H R^{\epsilon} \wedge g \wedge B\right)_{N, N-1} \wedge \varphi(\zeta)$. Hence,

$$
\begin{aligned}
\left(\int_{z}\left(H R^{\epsilon} \wedge g \wedge B\right)_{N, N-1}\right. & \wedge \bar{\partial} \mu(z)) \cdot \varphi(\zeta) \\
= & \pm \mu(z) \cdot\left(\bar{\partial}_{z} \int_{\zeta}\left(H R^{\epsilon} \wedge g \wedge B\right)_{N, N-1} \wedge \varphi(\zeta)\right)
\end{aligned}
$$

Similar formulas hold for the second and third term on the right-hand side of (3.1) and the lemma thus follows from Lemma 3.1.

Definition 3.3. - For a current $\mu$ in $D$ we let $\widetilde{P} \mu$ be the current in $D^{\prime}$ given by

$$
\widetilde{\mathscr{P}} \mu(\zeta)=\int_{z}(H R \wedge g)_{N, N} \wedge \mu(z)
$$

Notice, since $R=R(\zeta)$, that $(H R \wedge g)_{N, N} \wedge \mu(z)$ is well-defined as a tensor product in $D^{\prime} \times D$. Notice also that $\mathscr{P}$ maps arbitrary currents in $D$ to pseudomeromorphic currents in $D^{\prime}$ annihilated by $\mathcal{J}$.

The operator $\widetilde{\mathscr{P}}$ of Theorem 1.1 (and Theorem 4.3 below) corresponds to a choice of weight $g$ such that $\zeta \mapsto g(\zeta, z)$ is holomorphic, but in the definition above we do not require this.

Example 3.4. - Suppose that $D \subset \mathbb{C}^{N}$ is a ball and that $\mathcal{J}=\left\langle f_{1}, \ldots, f_{\kappa}\right\rangle$ is a complete intersection. Using the Koszul complex we get, see Example 2.1 and the notation there, that $R=R^{f} \wedge e_{1} \wedge \cdots \wedge e_{\kappa}$. Moreover, in view of the paragraph after Example 2.3 we get $H R=h_{1} \wedge \cdots \wedge h_{\kappa} \wedge R^{f} / \kappa$ !. Taking $g$ as in Example 2.2 with $\widetilde{s}=(2 \pi i)^{-1} \bar{z} \cdot \mathrm{d} \eta /\left(\zeta \cdot \bar{z}-|z|^{2}\right)$ and $\eta=\zeta-z$ we then get

$$
\widetilde{\mathscr{P}} \mu=R^{f} \wedge \int_{z} \frac{h_{1} \wedge \cdots \wedge h_{\kappa}}{\kappa !} \wedge \bar{\partial} \rho(z) \wedge \frac{(\bar{z} \cdot \mathrm{d} \eta) \wedge(\mathrm{d} \bar{z} \cdot \mathrm{d} \eta)^{N-\kappa-1}}{\left(2 \pi i\left(\zeta \cdot \bar{z}-|z|^{2}\right)\right)^{N-\kappa}} \wedge \mu(z) .
$$

Notice that, for degree reasons, $\widetilde{\mathscr{P}} \mu=0$ unless $\mu$ has degree $(*, \kappa)$.

Proposition 3.5. - Suppose that the weight $g$ in the definition of $\widetilde{P}$ depends holomorphically on $\zeta$. Then, for any $(p, q)$-current $\mu$ in $D, \widetilde{\mathscr{P}} \mu$ is of the form $\xi \cdot R_{q}^{0}$, where

$$
\xi=\Phi(\mu)=\int_{z} H_{q}^{0} \wedge g_{N-q} \wedge \mu(z)
$$


is a holomorphic p-form with values in $E_{q}^{*}$. Moreover, if $\mathcal{J} \mu=0$ then $\widetilde{\mathscr{P}} \bar{\partial} \mu=\bar{\partial} \widetilde{P}_{\mu}$ and $\Phi(\bar{\partial} \mu)=f_{q+1}^{*} \Phi(\mu)$, where $f_{q+1}^{*}$ is the transpose of the $\operatorname{map} f_{q+1}: E_{q+1} \rightarrow E_{q}$.

For the rest of this section we will fix frames for the trivial $E_{j}$-bundles and associated dual frames for the $E_{j}^{*}$ 's; sections of the $E_{j}$ 's will be represented by column vectors, sections of the $E_{j}^{*}$ 's by row vectors, and maps between bundles by matrices. Notice that $E_{0}$ is assumed to be the trivial line bundle so that $\operatorname{Hom}\left(E_{j}, E_{0}\right) \simeq E_{j}^{*}$. Recall that $f_{k}=f_{k}(\zeta)$. To prove Proposition 3.5 we will need

LEMMA 3.6. — Letting $(\cdot)^{*}$ denote matrix transpose we have

$$
f_{q+1}^{*}\left(H_{q}^{0}\right)^{*} \wedge g_{N-q}=\bar{\partial}\left(\left(H_{q+1}^{0}\right)^{*} \wedge g_{N-q-1}\right)+\left(f_{1}(z) H_{q+1}^{1}\right)^{*} \wedge g_{N-q} .
$$

Proof. - This is verified by the following computation.

$$
\begin{aligned}
f_{q+1}^{*}\left(H_{q}^{0}\right)^{*} \wedge g_{N-q} & =\left(H_{q}^{0} f_{q+1}\right)^{*} \wedge g_{N-q} \\
& =\left(\delta_{\eta} H_{q+1}^{0}+f_{1}(z) H_{q+1}^{1}\right)^{*} \wedge g_{N-q} \\
& =\left(\delta_{\eta} H_{q+1}^{0} \wedge g_{N-q}\right)^{*}+\left(f_{1}(z) H_{q+1}^{1}\right)^{*} \wedge g_{N-q} \\
& =\left( \pm H_{q+1}^{0} \wedge \delta_{\eta} g_{N-q}\right)^{*}+\left(f_{1}(z) H_{q+1}^{1}\right)^{*} \wedge g_{N-q} \\
& =\left( \pm H_{q+1}^{0} \wedge \bar{\partial} g_{N-q-1}\right)^{*}+\left(f_{1}(z) H_{q+1}^{1}\right)^{*} \wedge g_{N-q} \\
& =\bar{\partial}\left(H_{q+1}^{0} \wedge g_{N-q-1}\right)^{*}+\left(f_{1}(z) H_{q+1}^{1}\right)^{*} \wedge g_{N-q}
\end{aligned}
$$

where the second equality follows from the properties of the Hefer morphisms (see Example 2.3), the forth since $0=\delta_{\eta}\left(H_{q+1}^{0} \wedge g_{N-q}\right)$ for degree reasons, the fifth since $g$ is a weight, and the sixth since $H_{q+1}^{0}$ is holomorphic.

Proof of Proposition 3.5. - Since $g$ is holomorphic in $\zeta$ and in particular contains no $d \bar{\zeta}$-differentials, it follows for degree reasons that

$$
\widetilde{\mathscr{P}} \mu(\zeta)=\int_{z} H_{q}^{0} R_{q}^{0} \wedge g_{N-q} \wedge \mu(z)= \pm\left(R_{q}^{0}\right)^{*} \int_{z}\left(H_{q}^{0}\right)^{*} \wedge g_{N-q} \wedge \mu(z)
$$

Since also $H$ is holomorphic in $\zeta$ the first statement of the proposition follows. For the rest of this proof we will write $R_{j}$ instead of $R_{j}^{0}$. 
Assume that $\mathcal{J} \mu=0$. To see that $\widetilde{\mathscr{P}} \bar{\partial} \mu=\bar{\partial} \widetilde{\mathscr{P}} \mu$ we compute:

$$
\begin{aligned}
\widetilde{\mathscr{P}} \bar{\partial} \mu & =\int_{z} H_{q+1}^{0} R_{q+1} \wedge g_{N-q-1} \wedge \bar{\partial} \mu(z) \\
& = \pm R_{q+1}^{*} \int_{z} \bar{\partial}\left(\left(H_{q+1}^{0}\right)^{*} \wedge g_{N-q-1}\right) \wedge \mu(z) \\
& = \pm R_{q+1}^{*} \int_{z} f_{q+1}^{*}\left(H_{q}^{0}\right)^{*} \wedge g_{N-q} \wedge \mu(z) \\
& \mp R_{q+1}^{*} \int_{z}\left(f_{1}(z) H_{q+1}^{1}\right)^{*} \wedge g_{N-q} \wedge \mu(z) \\
& = \pm\left(f_{q+1} R_{q+1}\right)^{*} \int_{z}\left(H_{q}^{0}\right)^{*} \wedge g_{N-q} \wedge \mu(z) \\
& =\bar{\partial}\left(R_{q}^{*} \int_{z}\left(H_{q}^{0}\right)^{*} \wedge g_{N-q} \wedge \mu(z)\right)=\bar{\partial} \mathscr{P} \mu,
\end{aligned}
$$

where the second equality holds since $R=R(\zeta)$ is independent of $z$, the third by Lemma 3.6, the forth since (the entries of) $f_{1}(z)$ annihilate $\mu(z)$, and the fifth since $\nabla_{\text {End }} R=0$ (see Section 2.2) and $H$ and $g$ are holomorphic in $\zeta$.

Moreover, in view of Lemma 3.6, we get that

$$
\begin{aligned}
\Phi(\bar{\partial} \mu)^{*} & =\int_{z} \bar{\partial}\left(\left(H_{q+1}^{0}\right)^{*} \wedge g_{N-q-1}\right) \wedge \mu(z) \\
& =f_{q+1}^{*} \int_{z}\left(H_{q}^{0}\right)^{*} \wedge g_{N-q} \wedge \mu(z)+\int_{z}\left(f_{1}(z) H_{q+1}^{1}\right)^{*} \wedge g_{N-q} \wedge \mu(z) \\
& =f_{q+1}^{*} \Phi(\mu)^{*}
\end{aligned}
$$

since (the entries of) $f_{1}(z)$ annihilate $\mu(z)$.

Let $\mu \in \mathscr{P} \mathscr{M}(D)$. Since $B$ is almost semi-meromorphic in $D \times D$, the product $B \wedge \mu(z)$ is a well-defined pseudomeromorphic current in $D \times D$, in view of Section 2.1; by the uniqueness in [20, Theorem 5.1.1], $B \wedge \mu(z)$ coincides with the tensor product of $B$ and $\mu$ ( $z$ and $\zeta-z$ are independent variables). From Section 2.2, $R$ is an almost semi-meromorphic current plus $\bar{\partial}$ of such a current. Thus, by Section 2.1, $R \wedge B \wedge \mu(z)$ is a well-defined pseudomeromorphic current in $D^{\prime} \times D$ that can be defined as $\lim _{\epsilon \rightarrow 0} R^{\epsilon} \wedge B \wedge$ $\mu(z)$, where $R^{\epsilon}$ is the regularization of $R$ given by (2.3). Even though $R_{j}^{0}=0$ for $j<\kappa$ it may be the case that $\lim _{\epsilon \rightarrow 0} R_{j}^{0, \epsilon} \wedge B \wedge \mu(z) \neq 0$. Still, the support of $R \wedge B \wedge \mu(z)$ is contained in $Y \times \operatorname{supp} \mu$. To see this it suffices, in view of (2.3) and Section 2.1, to see that $\mathbf{1}_{Y \times D} B \wedge \mu(z)=0$. Since $B$ is smooth outside of the diagonal $\Delta \subset D \times D$ it is clear that supp $\mathbf{1}_{Y \times D} B \wedge \mu(z) \subset \Delta$. Moreover, $Z S S(B) \subset \Delta$ and so $\mathbf{1}_{\Delta} B \wedge \mu(z)=0$. Hence,

$$
\mathbf{1}_{Y \times D} B \wedge \mu(z)=\mathbf{1}_{\Delta} \mathbf{1}_{Y \times D} B \wedge \mu(z)=\mathbf{1}_{Y \times D} \mathbf{1}_{\Delta} B \wedge \mu(z)=0 .
$$


Definition 3.7. - For a pseudomeromorphic current $\mu$ in $D$ we let $\widetilde{\mathscr{K}} \mu$ be the pseudomeromorphic current in $D^{\prime}$ given by

$$
\widetilde{\mathscr{K}} \mu(\zeta)=\int_{z}(H R \wedge g \wedge B)_{N, N-1} \wedge \mu(z) .
$$

As in the definition of the $\widetilde{\mathscr{P}}$-operators, we do not require $g$ to be holomorphic in $\zeta$, but the $\widetilde{\mathscr{K}}$-operator of Theorem 4.3 corresponds to such a choice.

Notice that, since supp $R \wedge B \wedge \mu(z) \subset Y \times D, \widetilde{\mathscr{K}}$ maps pseudomeromorphic currents in $D$ to pseudomeromorphic currents in $D^{\prime}$ with support contained in $Y$. We do not know whether or not $\mathcal{J} \widetilde{\mathscr{K}} \mu=0$ for a general pseudomeromorphic $\mu$.

The following proposition follows from Lemma 3.2 by letting $\epsilon \rightarrow 0$.

Proposition 3.8. - For any $\mu \in \mathscr{P}_{\mathscr{M}^{p, q}}(D)$ such that $\mathcal{J} \mu=0$ we have $\mu_{\Gamma_{D^{\prime}}}=\bar{\partial} \widetilde{\mathscr{K}} \mu+\widetilde{\mathscr{K}} \bar{\partial} \mu+\widetilde{\mathscr{P}} \mu$.

Let $\mathscr{C} \mathscr{H}_{\mathcal{J}}^{p}$ be the subsheaf of $\mathscr{C}_{Y}^{p}$ of currents annihilated by $\mathcal{J}$.

Proposition 3.9. - Let $\widetilde{\mathscr{P}}$ be an operator associated to $\mathcal{J}$ and corresponding to a weight $g$ such that $\zeta \mapsto g(\zeta, z)$ is holomorphic. If $\nu \in \mathscr{C} \mathscr{H}_{\mathcal{J}}^{p}(D)$, then $\widetilde{P}_{\nu}=\nu_{\left.\right|_{D^{\prime}}}$. Moreover, if $\mu$ is any $\bar{\partial}$-closed $(p, \kappa)$-current such that $\mathcal{J} \mu=0$, then $\mathscr{P} \mu \in \mathscr{C}_{\mathcal{J}}^{p}\left(D^{\prime}\right)$ and $\widetilde{P}_{\mu}$ is the current $\nu$ in (1.1).

Proof. - By Proposition 3.8, $\nu_{\uparrow_{D^{\prime}}}=\bar{\partial} \widetilde{\mathscr{K}} \nu+\widetilde{\mathscr{P}} \nu$. However, $\widetilde{\mathscr{K}} \nu$ is a pseudomeromorphic $(p, \kappa-1)$-current with support contained in $Y$ and must thus vanish in view of the Dimension principle.

For the second statement, notice that $\widetilde{P}_{\mu}$ is a pseudomeromorphic $(p, \kappa)$ current annihilated by $\mathcal{J}$ (in particular with support in $Y$ ) and, by Proposition 3.5, $\bar{\partial} \widetilde{P}_{\mu}=0$. Thus, in view of Section 2.1, $\widetilde{P}_{\mu}$ is a section of $\mathscr{C} \mathscr{H}_{\mathcal{J}}^{p}$. Now consider the decomposition (1.1) and recall that $\mathcal{J} \nu=0$ and that we may assume that $\mathcal{J} \tau=0$. By the first part of the proof and Proposition 3.5

$$
\widetilde{\mathscr{P}} \mu=\widetilde{\mathscr{P}} \nu+\widetilde{\mathscr{P}} \bar{\partial} \tau=\nu+\bar{\partial} \widetilde{\mathscr{P}}_{\tau} \text {. }
$$

Together with (1.1) this gives that $\mu-\widetilde{\mathscr{P}}_{\mu}=\bar{\partial}\left(\tau-\widetilde{\mathscr{P}}_{\tau}\right)$, and so by uniqueness of (1.1), $\widetilde{\mathscr{P}}_{\mu}=\nu$.

Proof of Theorem 1.1. - Setting $P(\zeta, z):=H_{\kappa}^{0} \wedge g_{N-\kappa}$, where $g$ is a weight depending holomorphically on $\zeta$, Theorem 1.1 follows from Propositions 3.5 and 3.9. 


\section{Higher local moderate cohomology}

Let $\mathscr{C}_{\mathcal{J}}^{p, q}$ be the sheaf of $(p, q)$-currents in $M$ annihilated by $\mathcal{J}$. Notice that we have a complex $\left(\mathscr{C}_{\mathcal{J}}^{p, \bullet}, \bar{\partial}\right)$. The Dickenstein-Sessa decomposition (1.1) implies that there is a canonical isomorphism $\mathscr{H}^{\kappa}\left(\mathscr{C}_{\mathcal{J}}^{p}, \bar{\partial}\right) \simeq \mathscr{C}_{\mathcal{H}}^{p}$, where, as above, $\mathscr{C} \mathscr{H}_{\mathcal{J}}^{p}$ is the subsheaf of $\mathscr{C} \mathscr{H}_{Y}^{p}$ of currents annihilated by $\mathcal{J}$. The map $\mathscr{C} \mathscr{H}_{\mathcal{J}}^{p} \rightarrow \mathscr{H}^{\kappa}\left(\mathscr{C}_{\mathcal{J}}^{p, \bullet}, \bar{\partial}\right)$ is induced by the inclusion $\mathscr{C}_{\mathcal{J}}^{p} \hookrightarrow \mathscr{C}_{\mathcal{J}}^{p, \kappa}$ and the inverse map $\mathscr{H}^{\kappa}\left(\mathscr{C}_{\mathcal{J}}^{p, \bullet}, \bar{\partial}\right) \rightarrow \mathscr{C}_{\mathcal{H}_{\mathcal{J}}}^{p}$ is induced by our operator $\mathscr{P}$. It is well-known that $\mathscr{H}^{q}\left(\mathscr{C}_{\mathcal{J}}^{p, \bullet}, \bar{\partial}\right)=0$ for $q<\kappa$, see, e.g., [13]. Our objective now is to give a description of the higher cohomology $\mathscr{H}^{q}\left(\mathscr{C}_{\mathcal{J}}^{p, \bullet}, \bar{\partial}\right), q>\kappa$.

Remark 4.1. - It is well-known that the cohomology sheaves $\mathscr{H}^{q}\left(\mathscr{C}_{\mathcal{J}}^{p, \bullet}, \bar{\partial}\right)$ are isomorphic to $\mathscr{E}_{x t^{q}}\left(\mathscr{O}_{M} / \mathcal{J}, \Omega_{M}^{p}\right)$, where $\Omega_{M}^{p}$ is the sheaf of holomorphic $p$-forms on $M$.

THEOREM 4.2. - There are fine sheaves of currents $\mathscr{B}_{\mathcal{J}}^{p, q} \subset \mathscr{C}_{\mathcal{J}}^{p, q}, p \geqslant 0$, $q \geqslant \kappa$, such that $\bar{\partial}$ maps $\mathscr{B}_{\mathcal{J}}^{p, q}$ to $\mathscr{B}_{\mathcal{J}}^{p, q+1}$ and the inclusion $\mathscr{B}_{\mathcal{J}}^{p, q} \subset \mathscr{C}_{\mathcal{J}}^{p, q}$ induces an isomorphism $\mathscr{H}^{q}\left(\mathscr{B}_{\mathcal{J}}^{p, \bullet}, \bar{\partial}\right) \simeq \mathscr{H}^{q}\left(\mathscr{C}_{\mathcal{J}}^{p, \bullet}, \bar{\partial}\right)$. Setting $\mathscr{B}_{\mathcal{J}}^{p, q}=0$ for $q<\kappa$ this holds for all $q$. Moreover, $\overline{\sqrt{\mathcal{J}}} \mathscr{B}_{\mathcal{J}}^{p, q}=d \overline{\sqrt{\mathcal{J}}} \wedge \mathscr{B}_{\mathcal{J}}^{p, q}=0$.

The point is that $\mathscr{B}_{\mathcal{J}}^{p, q}$ is much smaller and less singular than $\mathscr{C}_{\mathcal{J}}^{p, q}$; cf. Example 4.5 below. Moreover, $\mathscr{B}_{\mathcal{J}}^{p, q}$ is a concretely defined subsheaf of $\mathscr{P} \mathscr{M}_{M}^{p, q}$, and the kernel of $\bar{\partial}$ in $\mathscr{B}_{\mathcal{J}}^{p, \kappa}$ is $\mathscr{C}_{\mathcal{J}}^{p}$.

Theorem 4.2 shows that the natural inclusion of complexes $\left(\mathscr{B}_{\mathcal{J}}^{p, \bullet}, \bar{\partial}\right) \hookrightarrow$ $\left(\mathscr{C}_{\mathcal{J}}^{p, \bullet}, \bar{\partial}\right)$ is a quasi-isomorphism, i.e., an isomorphism on cohomology. Our final result provides in particular an explicit projection operator $\widetilde{\mathscr{P}}:\left(\mathscr{C}_{\mathcal{J}}^{p}, \bar{\partial}\right) \rightarrow$ $\left(\mathscr{B}_{\mathcal{J}}^{p}, \bar{\partial}\right)$ giving the inverse of this quasi-isomorphism. Let $\mathscr{C}^{p, q}$ be the sheaf of $(p, q)$-currents in $M$.

TheOREM 4.3. - Let $M \subset \mathbb{C}^{N}$ be pseudoconvex and let $M^{\prime} \Subset M$. There is an integral operator $\widetilde{\mathscr{P}}_{:} \mathscr{C}^{p, q}(M) \rightarrow \mathscr{B}_{\mathcal{J}}^{p, q}\left(M^{\prime}\right)$ giving a quasi-isomorphism of complexes $\left(\mathscr{C}_{\mathcal{J}}^{p, \bullet}, \bar{\partial}\right) \rightarrow\left(\mathscr{B}_{\mathcal{J}}^{p, \bullet} \bar{\partial}\right)$. Moreover, there is an integral operator $\widetilde{\mathscr{K}}: \mathscr{B}_{\mathcal{J}}^{p, q}(M) \rightarrow \mathscr{B}_{\mathcal{J}}^{p, q-1}\left(M^{\prime}\right)$ such that $\mu_{\uparrow_{M^{\prime}}}=\bar{\partial} \widetilde{\mathscr{K}} \mu+\widetilde{\mathscr{K}} \bar{\partial} \mu+\mathscr{P}_{\mu}$ for any $\mu \in \mathscr{B}_{\mathcal{J}}^{p, q}(M)$.

In analogy with [8, Definition 7.1], [23, Definition 4.1], and [24, Section 6.2$]$ we make the following definition.

Definition 4.4. - A $(p, q)$-current $\mu$ on $M$ is a section of $\mathscr{B}_{\mathcal{J}}^{p, q}$ over an open set $\mathcal{U} \subset M$ if for each $x \in \mathcal{U}$ there is a neighborhood $D$ of $x$ such 
that $\mu_{\uparrow_{D}}$ is a finite sum of currents of the form

$$
\xi_{m} \wedge \widetilde{\mathscr{K}}_{m}\left(\cdots \xi_{1} \wedge \widetilde{\mathscr{K}}_{1}\left(\xi_{0} \cdot R\right) \cdots\right),
$$

where $R$ is a current corresponding to a Hermitian free resolution $\left(\mathscr{O}\left(E_{\bullet}\right), f_{\bullet}\right)$ of $\mathscr{O} / \mathcal{J}$ in $D, \xi_{0}$ is a smooth form with values in $\bigoplus_{q} E_{q}^{*}, \xi_{j}$ is a smooth form for $j \geqslant 1$, and the $\widetilde{\mathscr{K}_{j}}$ 's are operators as in Definition 3.7.

It is clear from the definition that $\mathscr{B}_{\mathcal{J}}:=\bigoplus_{p, q} \mathscr{B}_{\mathcal{J}}^{p, q}$ is a module over the sheaf $\bigoplus_{p, q} \mathscr{E}_{M}^{p, q}$ of smooth forms on $M$ and that it is closed under $\widetilde{\mathscr{K}}$ operators. Moreover, $\mathscr{B}_{\mathcal{J}} \subset \mathscr{P} \mathscr{M}$ and any section of $\mathscr{B}_{\mathcal{J}}$ has support in $Y$. Hence, in view of Section 2.1, if $\mu \in \mathscr{B}_{\mathcal{J}}^{p, \kappa}$ is $\bar{\partial}$-closed then $\mu \in \mathscr{C}_{\mathscr{H}_{Y}}^{p}$. Notice also, by Section 3 , that a $\widetilde{\mathscr{P}}$-operator associated with $\mathcal{J}$ maps an arbitrary current to a section of $\mathscr{B}_{\mathcal{J}}$.

Example 4.5. - Let $i: Y \hookrightarrow M$, suppose that $Y$ is smooth and that $\mathcal{J}$ is the radical ideal sheaf of $Y$. We claim that then $\mathscr{B}_{\mathcal{J}}^{N, q}=i_{*} \mathscr{E}_{Y}^{N-\kappa, q-\kappa}$. Given the claim, we see that

$$
0 \longrightarrow \mathscr{B}_{\mathcal{J}}^{N, \kappa} \stackrel{\bar{\partial}}{\longrightarrow} \cdots \stackrel{\bar{\partial}}{\longrightarrow} \mathscr{B}_{\mathcal{J}}^{N, N} \longrightarrow 0
$$

is $i_{*}$ of the Dolbeault complex of smooth $(\operatorname{dim} Y, \bullet)$-forms on $Y$. Moreover, since, by Theorem 4.2 , the kernel of $\bar{\partial}$ at $\mathscr{B}_{\mathcal{J}}^{N, \kappa}$ is $\mathscr{C} \mathscr{H}_{\mathcal{J}}^{N}$ it follows that $i_{*} \Omega_{Y}^{\operatorname{dim} Y}=\mathscr{C} \mathscr{H}_{\mathcal{J}}^{N}$.

The claim may be verified locally so we assume that $M$ is the unit ball in $\mathbb{C}^{N}$ with coordinates $(z ; w)$ such that $Y=\{z=0\}$ and $\mathcal{J}=\langle z\rangle$. For notational convenience we will also assume that $\kappa=1$. Let $\varphi$ be a smooth $(N-1, q-1)$-form on $Y$ and let $\widetilde{\varphi}$ be any smooth extension of $\varphi$ to $M$. Then $i_{*} \varphi=\tilde{\varphi} \wedge[Y]$. From the free resolution $0 \rightarrow \mathscr{O} \stackrel{z}{\rightarrow} \mathscr{O}$ of $\mathscr{O} / \mathcal{J}$ we get the current $R=\bar{\partial}(1 / z)$ and so, by the Poincaré-Lelong formula, $\widetilde{\varphi} \wedge[Y]=i \widetilde{\varphi} \wedge \mathrm{d} z \wedge R / 2 \pi$. Hence, $i_{*} \mathscr{E}_{Y}^{N-\kappa, q-\kappa} \subset \mathscr{B}_{\mathcal{J}}^{N, q}$.

For the opposite inclusion, let $\mu$ be a section of $\mathscr{B}_{\mathcal{J}}^{N, q}$; we may assume that $\mu$ is of the form (4.1). We use induction over $m$ in (4.1) to see that $\mu=\xi \wedge[Y]$ for some smooth $\xi$; notice that such a $\xi$ exists if there is a continuous $\xi^{\prime}$, which is smooth along $Y$, such that $\mu=\xi^{\prime} \wedge[Y]$. If $\left(\mathscr{O}\left(E_{\bullet}\right), f_{\bullet}\right)$ is any free Hermitian resolution of $\mathscr{O} / \mathcal{J}$ with rank $E_{0}=1$, then the associated residue current is of the form $\alpha \wedge \bar{\partial}(1 / z)$, where $\alpha$ is a smooth $(0, *)$-form with values in $\bigoplus_{j} E_{j}$, see [9, Theorem 4.4]. Hence, if $\mu$ is of the form (4.1) with $m=0$, then $\mu=\xi_{0} \cdot \alpha \wedge \bar{\partial}(1 / z)$, where $\xi_{0}$ is a smooth $(N, *)$-form with values in $\bigoplus_{j} E_{j}^{*}$. Writing $\xi_{0} \cdot \alpha=\xi \wedge d z$, for some scalar-valued smooth form $\xi$, we get $\xi_{0} \cdot \alpha \wedge \bar{\partial}(1 / z)=-2 \pi i \xi \wedge[Y]$, and the induction start follows. Now, if 
$\widetilde{\mathscr{K}}$ is an integral operator as in Definition 3.7, then

$$
\widetilde{\mathscr{K}}(\xi \wedge[Y])=\int_{z \in Y}(H R \wedge g \wedge B)_{N, N-1} \wedge \xi(z)=R \cdot \int_{z \in Y} k(\zeta, z) \wedge \xi(z),
$$

where $R=R(\zeta)$ is of the form $\alpha(\zeta) \wedge \bar{\partial}(1 / \zeta)$ as above, and $k(\zeta, z)$ is a kernel in $M \times M$ which is $\mathcal{O}\left(1 /|\zeta-z|^{2(N-1)-1}\right)$. It follows that $\zeta \mapsto \int_{z \in Y} k(\zeta, z) \wedge \xi(z)$ is a continuous $(N, *)$-form with values in $\bigoplus_{j} E_{j}^{*}$ and we write it as $\psi(\zeta) \wedge d \zeta$. Moreover, in view of [8, Lemma 6.2], $\psi_{\uparrow_{Y}}$ is smooth. We get

$$
\widetilde{\mathscr{K}}(\xi \wedge[Y])=\alpha \wedge \bar{\partial}(1 / \zeta) \cdot \psi \wedge d \zeta= \pm 2 \pi i \alpha \cdot \psi \wedge[Y],
$$

and the induction step follows.

Remark 4.6. - For not necessarily smooth $Y$ and any $\mathcal{J}$ of pure codimension $\kappa$ and zero set $Y$, Andersson and Lärkäng introduce a notion of holomorphic top-degree forms on the possibly non-reduced complex space $Y_{\mathcal{J}}=\left(Y, \mathscr{O}_{M} / \mathcal{J}\right)$, see $\left[7\right.$, Section 5]; see [12] for the reduced case. Via $i_{*}$ this notion precisely corresponds to $\mathscr{C} \mathscr{H}_{\mathcal{J}}^{N}$. By analogy it is reasonable to think of (4.2) as a certain Dolbeault complex for $Y_{\mathcal{J}}$ with the cohomology at $\mathscr{B}_{\mathcal{J}}^{N, \kappa}$ being the holomorphic top-degree forms on $Y_{\mathcal{J}}$ and $\widetilde{P}$ as a projection operator onto these forms.

To prove Theorem 4.2 (and 4.3) we may assume that $M$ and $M^{\prime}$ are as in Theorem 4.3. Let $M^{\prime \prime} \Subset D$ be a suitable neighborhood of $\bar{M}^{\prime}$ similar to the beginning of Section 3. Let

$$
0 \longrightarrow \mathscr{O}\left(E_{m}\right) \stackrel{f_{m}}{\longrightarrow} \cdots \stackrel{f_{1}}{\longrightarrow} \mathscr{O}\left(E_{0}\right) \longrightarrow \mathscr{O} / \mathcal{J} \longrightarrow 0
$$

be a Hermitian free resolution of $\mathscr{O} / \mathcal{J}$ in $M^{\prime \prime}$ and let $U$ and $R$ be the associated currents, see Section 2.2. Dualizing and tensoring with $\Omega^{p}:=\Omega_{M}^{p}$ we get the complex

$$
0 \longleftarrow \mathscr{O}\left(E_{m}^{*}\right) \otimes \Omega^{p} \stackrel{f_{m}^{*} \otimes \mathrm{Id}}{\longleftarrow} \cdots \stackrel{f_{1}^{*} \otimes \mathrm{Id}}{\longleftarrow} \mathscr{O}\left(E_{0}\right) \otimes \Omega^{p} \longleftarrow 0 .
$$

It is well-known that the cohomology of this complex is isomorphic to $\mathscr{E} x t^{\bullet}\left(\mathscr{O}_{M} / \mathcal{J}, \Omega^{p}\right)$. Recall that $\mathscr{C}^{p, q}$ is a stalk-wise injective $\mathscr{O}_{M}$-module by a well-known theorem of Malgrange and consider the resolution

$$
0 \longrightarrow \Omega^{p} \longrightarrow \mathscr{C}^{p, 0} \stackrel{\bar{\partial}}{\longrightarrow} \cdots \stackrel{\bar{\partial}}{\longrightarrow} \mathscr{C}^{p, N} \longrightarrow 0
$$

of $\Omega^{p}$. Applying the functor $\mathscr{H}$ om $\left(\mathscr{O}_{M} / \mathcal{J},-\right)$ and noticing that $\mathscr{H}$ om $\left(\mathscr{O}_{M} / \mathcal{J}, \mathscr{C}^{p, q}\right) \simeq \mathscr{C}_{\mathcal{J}}^{p, q}$ we get the complex

$$
0 \longrightarrow \mathscr{C}_{\mathcal{J}}^{p, 0} \stackrel{\bar{\partial}}{\longrightarrow} \cdots \stackrel{\bar{\partial}}{\longrightarrow} \mathscr{C}_{\mathcal{J}}^{p, N} \longrightarrow 0
$$

By standard homological algebra, the cohomology of (4.4) is naturally isomorphic to the cohomology of (4.3). Following [6] this isomorphism can be realized using the current $R$ as follows. For convenience we will write just $f_{k}^{*}$ 
instead of $f_{k}^{*} \otimes$ Id. If $\xi$ is a holomorphic $p$-form with values in $E_{q}^{*}$ such that $f_{q+1}^{*} \xi=0$, then since $f R-\bar{\partial} R=0$ (see Section 2.2)

$$
\bar{\partial}\left(\xi \cdot R_{q}\right)= \pm \xi \cdot \bar{\partial} R_{q}= \pm \xi \cdot f_{q+1} R_{q+1}= \pm f_{q+1}^{*} \xi \cdot R_{q+1}=0 .
$$

Similarly, if $\xi=f_{q}^{*} \xi^{\prime}$, then $\xi \cdot R_{q}=0$. Thus the map $\mathscr{O}\left(E_{p}^{*}\right) \otimes \Omega^{p} \rightarrow \mathscr{C}_{\mathcal{J}}^{p, q}$ given by $\xi \mapsto \xi \cdot R_{q}$ induces a map on cohomology and it turns out to be the natural isomorphism. Notice that $\xi \cdot R_{q}$ is a section of $\mathscr{B}_{\mathcal{J}}^{p, q}$.

Proof of Theorems 4.2 and 4.3. - We have already noticed that any $\widetilde{P}$-operator associated to $\mathcal{J}$ maps arbitrary currents to sections of $\mathscr{B}_{\mathcal{J}}$ and that any $\widetilde{\mathscr{K}}$-operator associated to $\mathcal{J}$ maps sections of $\mathscr{B} \mathcal{J}$ to sections of $\mathscr{B}_{\mathcal{J}}$. As $\mathscr{B}_{\mathcal{J}} \subset \mathscr{P} \mathscr{M}$ and sections of $\mathscr{B}_{\mathcal{J}}$ have support in $Y$ it follows from the Dimension principle that $\overline{\sqrt{\mathcal{J}}} \mathscr{B}_{\mathcal{J}}=\mathrm{d} \overline{\sqrt{\mathcal{J}}} \wedge \mathscr{B}_{\mathcal{J}}=0$.

Let us temporarily assume that $\mathcal{J} \mathscr{B}_{\mathcal{J}}^{p, q}=0$ and show how Theorems 4.2 and 4.3 follow. Then the kernel of $\bar{\partial}$ in $\mathscr{B}_{\mathcal{J}}^{p, \kappa}$ is $\mathscr{C}_{\mathscr{H}}^{p}$ and, by Proposition 3.8, we have $\mu=\bar{\partial} \widetilde{\mathscr{K}} \mu+\widetilde{\mathscr{K}} \bar{\partial} \mu+\widetilde{\mathscr{P}} \mu$ for any $\mu$ in $\mathscr{B}_{\mathcal{J}}$ if $\widetilde{\mathscr{P}}$ and $\widetilde{\mathscr{K}}$ are constructed using the same $R, H$, and $g$. Assume henceforth that $g$ depends holomorphically on $\zeta$.

To show that $\bar{\partial}$ maps $\mathscr{B}_{\mathcal{J}}$ to itself it suffices to show that if $\mu$ is of the form (4.1) (with $\xi_{m}=1$ ) then $\bar{\partial} \mu$ is a section of $\mathscr{B}_{\mathcal{J}}$; we will use induction over $m$ to see this. The case $m=0$ follows since $\nabla_{\text {End }} R=0$. Indeed, then $\mu=\xi \cdot R$ for some smooth $\xi$ and a computation similar to (4.5) gives

$$
\bar{\partial}(\xi \cdot R)=\bar{\partial} \xi \cdot R \pm f^{*} \xi \cdot R .
$$

If $m>0$ we write $\mu=\widetilde{\mathscr{K}_{m}} \mu^{\prime}$, where $\mu^{\prime}$ is of the form (4.1) with $m$ replaced by $m-1$. By Proposition 3.8 we get

$$
\bar{\partial} \mu=\bar{\partial} \widetilde{\mathscr{K}}_{m} \mu^{\prime}=\mu^{\prime}-\widetilde{\mathscr{K}}_{m} \bar{\partial} \mu^{\prime}-\widetilde{\mathscr{P}}_{m} \mu^{\prime},
$$

and since $\bar{\partial} \mu^{\prime}$ is a section of $\mathscr{B}_{\mathcal{J}}$ by the induction hypothesis, it follows that $\bar{\partial} \mu$ is a section of $\mathscr{B}_{\mathcal{J}}$.

To see that the inclusion of complexes $\left(\mathscr{B}_{\mathcal{J}}^{p, \bullet}, \bar{\partial}\right) \hookrightarrow\left(\mathscr{C}_{\mathcal{J}}^{p, \bullet}, \bar{\partial}\right)$ is a quasiisomorphism, consider the diagram

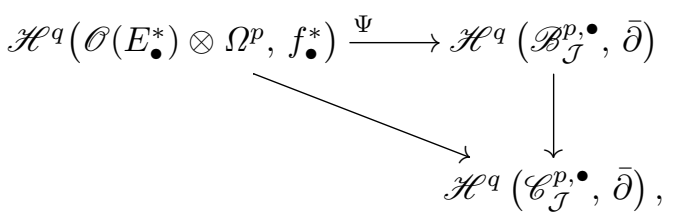

where the diagonal map is the natural isomorphism and $\Psi$ is the map $\xi \mapsto$ $\xi \cdot R_{q}$; cf. [6, Section 7]. It follows that $\Psi$ is injective and that the vertical 
map is surjective. Let $\mu$ be a $\bar{\partial}$-closed section of $\mathscr{B}_{\mathcal{J}}^{p, q}$ and suppose that there is a $\tau \in \mathscr{C}_{\mathcal{J}}^{p, q-1}$ such that $\mu=\bar{\partial} \tau$. Then by Propositions 3.8 and 3.5

$$
\mu=\bar{\partial} \widetilde{\mathscr{K}} \mu+\widetilde{\mathscr{P}} \mu=\bar{\partial}(\widetilde{\mathscr{K}} \mu+\widetilde{\mathscr{P}} \tau) \text {. }
$$

Since $\widetilde{\mathscr{K}} \mu+\widetilde{\mathscr{P}}_{\tau}$ is in $\mathscr{B}_{\mathcal{J}}$ it follows that the vertical map is injective. Thus, the horizontal map must be surjective and so all maps in (4.6) are isomorphisms.

Notice that $\bar{\partial} \widetilde{P} \mu=\widetilde{\mathscr{P}} \bar{\partial} \mu$ for any $\mu \in \mathscr{B}_{\mathcal{J}}$ by Proposition 3.5. Hence, $\widetilde{\mathscr{P}}$ is a map of complexes $\left(\mathscr{C}_{\mathcal{J}}^{p, \bullet}, \bar{\partial}\right) \rightarrow\left(\mathscr{B}_{\mathcal{J}}^{p, \bullet}, \bar{\partial}\right)$. Showing that it induces the inverse of the vertical map in (4.6) is similar to the proof of Proposition 3.9: Let $\mu \in \mathscr{C}_{\mathcal{J}}^{p, q}$ be $\bar{\partial}$-closed; we must show that $\widetilde{\mathscr{P}} \mu$ is a $\bar{\partial}$-closed section of $\mathscr{B}_{\mathcal{J}}^{p, q}$ such that $\mu-\widetilde{\mathscr{P}} \mu \in \bar{\partial} \mathscr{C}_{\mathcal{J}}^{p, q-1}$. In view of Proposition 3.5 the first part of this is clear. The vertical map in (4.6) is an isomorphism and so there is a $\bar{\partial}$-closed $\nu \in \mathscr{B}_{\mathcal{J}}^{p, q}$ and a $\tau \in \mathscr{C}_{\mathcal{J}}^{p, q-1}$ such that (1.1) holds. Then, by Proposition 3.8, $\nu=\widetilde{\mathscr{P}} \nu+\bar{\partial} \widetilde{\mathscr{K}} \nu$ and so by Proposition 3.5 we get

$$
\widetilde{\mathscr{P}} \mu=\widetilde{\mathscr{P}} \nu+\widetilde{\mathscr{P}} \bar{\partial} \tau=\nu-\bar{\partial} \widetilde{\mathscr{K}} \nu+\bar{\partial} \widetilde{\mathscr{P}}_{\tau}
$$

by applying $\widetilde{\mathscr{P}}$ to (1.1). Subtracting this from (1.1) we get

$$
\mu-\widetilde{\mathscr{P}}_{\mu}=\bar{\partial}\left(\tau-\widetilde{\mathscr{P}}_{\tau}+\widetilde{\mathscr{K}} \nu\right) \text {. }
$$

To conclude the proof of Theorems 4.2 and 4.3 it remains to show that $\mathcal{J} \mu=0$ for any section $\mu$ of $\mathscr{B}_{\mathcal{J}}^{p, q}$. We may assume that $\mu$ is of the form (4.1). In view of Section 2.1, the product

$T_{m, q}:=R_{k_{m}}\left(z_{m}\right) \wedge B_{\ell_{m}}\left(z_{m}, z_{m-1}\right) \wedge \cdots \wedge B_{\ell_{1}}\left(z_{1}, z_{0}\right) \wedge R_{q}\left(z_{0}\right), \quad k_{j}+\ell_{j} \leqslant N$, is a well-defined pseudomeromorphic current in $M_{z_{0}}^{\prime \prime} \times \cdots \times M_{z_{m}}^{\prime \prime}$; here the $R$ 's are currents corresponding to Hermitian resolutions of $\mathscr{O} / \mathcal{J}$ and the $B$ 's are Bochner-Martinelli type forms as in Section $2.3 ; R_{k_{j}}$ is a component of the part of the $j^{\text {th }} R$-current of bidegree $\left(0, k_{j}\right)$ and $B_{\ell_{j}}$ is the part of the $j^{\text {th }}$ Bochner-Martinelli type form of bidegree $\left(\ell_{j}, \ell_{j}-1\right)$. In view of Definition 3.7, $\mu$ is a sum of push-forwards, under maps $M_{z_{0}}^{\prime \prime} \times \cdots \times M_{z_{m}}^{\prime \prime} \rightarrow$ $M_{z_{m}}^{\prime \prime}$, of $T_{m, q}$-currents times smooth forms, and therefore it is sufficient to show that $\mathcal{J} T_{m, q}=0$ where $\mathcal{J}=\mathcal{J}\left(z_{m}\right)$; we will do this by double induction over $m$ and $q$. Since $T_{0, q}=R_{q}$ it is clear that $\mathcal{J} T_{0, q}=0$ for all $q$. Fix now $m>0$ and notice that $T_{m, q}$ has bidegree $\left(\sum_{j=1}^{m} \ell_{j}, \sum_{j=1}^{m} k_{j}+\ell_{j}-m+q\right)$ and that $\sum_{j=1}^{m} k_{j}+\ell_{j}-m+q \leqslant m N-m+q$. From the paragraph preceding Definition 3.7 it follows that the support of $T_{m, q}$ is contained in $Y \times \cdots \times Y$ $\left(m+1\right.$ copies). Moreover, if $z_{s+1} \neq z_{s}$ for some $0 \leqslant s \leqslant m-1$, then $B_{\ell_{s+1}}\left(z_{s+1}, z_{s}\right)$ is smooth and so $T_{m, q}$ is a smooth form times the tensor product of two currents $T_{s, q}$ and $T_{m-s-1, k_{s+1}}$. By induction over $m$ we have $\mathcal{J} T_{m-s-1, k_{s+1}}=0$. It follows that the support of $\mathcal{J} T_{m, q}$ is contained in 
$\left\{z_{0}=\cdots=z_{m}\right\} \cap Y \times \cdots \times Y \simeq Y$, which has codimension $m N+\kappa$ in $M_{z_{0}}^{\prime \prime} \times \cdots \times M_{z_{m}}^{\prime \prime}$. Thus, by the Dimension principle, $\mathcal{J} T_{m, q}=0$ if $m N-$ $m+q<m N+\kappa$, i.e., if $q<\kappa+m$. In particular, $\mathcal{J} T_{m, \kappa}=0$, which is the induction start for showing $\mathcal{J} T_{m, q}=0$ by induction over $q$. Let $R=R\left(z_{0}\right)=$ $R_{\kappa}\left(z_{0}\right)+R_{\kappa+1}\left(z_{0}\right)+\cdots$ be the $R$-current associated to the Hermitian free resolution $\left(\mathscr{O}\left(E_{\bullet}\right), f_{\bullet}\right)$ as in Section 2.2. From (the proof of) [9, Theorem 4.4] it follows that outside of the set $Y_{k}$ where $f_{k}$ does not have optimal rank, there is a $\operatorname{smooth} \operatorname{Hom}\left(E_{k}, E_{k+1}\right)$-valued $(0,1)$-form $\alpha_{k}=\alpha_{k}\left(z_{0}\right)$ such that $R_{k}=\alpha_{k} R_{k-1}$. Moreover, since $\left(\mathscr{O}\left(E_{\bullet}\right), f_{\bullet}\right)$ is exact, it follows from the Buchsbaum-Eisenbud criterion that $\operatorname{codim} Y_{k} \geqslant k, k \geqslant 1$. Hence, for $z_{0} \notin$ $Y_{\kappa+1}$ we have $T_{m, \kappa+1}=\alpha_{\kappa+1} T_{m, \kappa}$ and so the support of $\mathcal{J} T_{m, \kappa+1}$ must be contained in the set where $z_{0} \in Y_{\kappa+1}$. Since it also has support contained in $\left\{z_{0}=\cdots=z_{m}\right\}$ it must in fact have support contained in $\left\{z_{0}=\cdots=\right.$ $\left.z_{m}\right\} \cap Y_{\kappa+1} \times \cdots \times Y_{\kappa+1} \simeq Y_{\kappa+1}$, which has codimension $\geqslant m N+\kappa+1$ in $M_{z_{0}}^{\prime \prime} \times \cdots \times M_{z_{m}}^{\prime \prime}$. By the Dimension principle, then, $\mathcal{J} T_{m, \kappa+1}=0$. Continuing in this way we get that $\mathcal{J} T_{m, q}=0$ for all $q$.

The proof shows that the map $\Psi$ of (4.6) is an isomorphism. The injectivity followed since the diagonal map in (4.6) is an isomorphism which in turn relies on Malgrange's result that $\mathscr{C}^{p, q}$ is stalk-wise injective. However, both surjectivity and injectivity of $\Psi$ can be showed directly using the methods of this paper. To conclude the paper we sketch how this can be done. For the surjectivity of $\Psi$, let $\mu$ be a $\bar{\partial}$-closed section of $\mathscr{B}_{\mathcal{J}}^{p, q}$. Then, by Propositions 3.8 and $3.5, \mu=\xi \cdot R_{q}^{0}+\bar{\partial} \widetilde{\mathscr{K}} \mu$, where $f_{q+1}^{*} \xi=0$, and so the germ of a section of $\mathscr{H}^{q}\left(\mathscr{B}_{\mathcal{J}}^{p, \bullet}, \bar{\partial}\right)$ defined by $\mu$ is in the image of $\Psi$.

For the injectivity we will use a new kind of weight in our integral formulas to see that the map $\Phi$, defined in Proposition 3.5, induces a left inverse of $\Psi$. Notice that $\Phi$ indeed induces a map on cohomology since $\Phi(\bar{\partial} \mu)=f_{q+1}^{*} \Phi(\mu)$ for sections $\mu$ of $\mathscr{B}_{\mathcal{J}}^{p, q}$. With the setup of Example 2.3, consider

$$
\begin{aligned}
\check{G}_{q}^{\epsilon} & :=\sum_{\ell=0}^{q} R_{q}^{\ell, \epsilon}(z) H_{q}^{\ell}+\sum_{\ell=0}^{q-1} U_{q}^{\ell, \epsilon}(z) H_{q-1}^{\ell} f_{q}+\sum_{\ell=0}^{q} f_{q+1}(z) U_{q+1}^{\ell, \epsilon}(z) H_{q}^{\ell} \\
& =: R_{q}^{\epsilon}(z) H_{q}+U_{q}^{\epsilon}(z) H_{q-1} f_{q}+f_{q+1}(z) U_{q+1}^{\epsilon}(z) H_{q},
\end{aligned}
$$

which is a smooth section of $\Lambda_{\eta} \otimes \operatorname{Hom}\left(E_{q}^{\zeta}, E_{q}^{z}\right)$ for any $\epsilon>0$; notice that $U^{\epsilon}(z)$ and $R^{\epsilon}(z)$ here depend on $z$. One can check that $\check{G}_{q}^{\epsilon}$ satisfies the properties of being a weight, with the property $\breve{G}_{q, 0}^{\epsilon}(z, z)=1$ construed as $\breve{G}_{q, 0}^{\epsilon}(z, z)=\operatorname{Id}_{E_{q}}$. Let also $g=g(\zeta, z)$ be a suitable weight such that $\zeta \mapsto g(\zeta, z)$ is holomorphic and $z \mapsto g(\zeta, z)$ has compact support, cf. Example 2.2. Identifying sections of the $E_{j}^{*}$ 's with row vectors, sections of the $E_{j}$ 's with column vectors, mappings with matrices, and letting $(\cdot)^{*}$ denote matrix 
transpose as in Section 3, we get for any $E_{q}^{*}$-valued holomorphic $p$-form $\xi$, in view of Section 2.3, that

$$
\begin{aligned}
\xi^{*}(\zeta)= & \int_{z}\left(\breve{G}^{\epsilon} \wedge g\right)_{N, N}^{*} \wedge \xi^{*}(z) \\
= & \int_{z}\left(R_{q}^{\epsilon}(z) H_{q} \wedge g\right)_{N, N}^{*} \wedge \xi^{*}(z)+\int_{z}\left(U_{q}^{\epsilon}(z) H_{q-1} f_{q} \wedge g\right)_{N, N}^{*} \wedge \xi^{*}(z) \\
& +\int_{z}\left(f_{q+1}(z) U_{q+1}^{\epsilon}(z) H_{q} \wedge g\right)_{N, N}^{*} \wedge \xi^{*}(z) .
\end{aligned}
$$

Notice that the last integral vanishes if $f_{q+1}^{*} \xi^{*}=0$, and that the second last integral is $f_{q}^{*}$-exact. Since $R=R_{\kappa}^{0}+R_{\kappa+1}^{0}+\cdots$, it follows that $R_{q}^{\epsilon}(z) H_{q} \rightarrow$ $R_{q}^{0}(z) H_{q}^{0}$ as $\epsilon \rightarrow 0$, and so we see that if $f_{q+1}^{*} \xi^{*}=0$, then

$$
\xi^{*}(\zeta)=\int_{z}\left(R_{q}^{0}(z) H_{q}^{0} \wedge g_{N-q}\right)^{*} \wedge \xi^{*}(z)+f_{q}^{*} \widetilde{\xi}^{*}=\Phi\left(\xi R_{q}^{0}\right)^{*}+f_{q}^{*} \widetilde{\xi}^{*}
$$

where $\widetilde{\xi}$ is an $E_{q-1}^{*}$-valued holomorphic $p$-form. Hence, $\Phi$ induces a left inverse of $\Psi$.

\section{Bibliography}

[1] M. Andersson, "Integral representation with weights. I", Math. Ann. 326 (2003), no. 1 , p. 1-18.

[2] - "Residue currents and ideals of holomorphic functions", Bull. Sci. Math. 128 (2004), no. 6, p. 481-512.

[3] - "Residues of holomorphic sections and Lelong currents", Ark. Mat. 43 (2005), no. 2, p. 201-219.

[4] - "Integral representation with weights. II. Division and interpolation", Math. Z. 254 (2006), no. 2, p. 315-332.

[5] - "Uniqueness and factorization of Coleff-Herrera currents", Ann. Fac. Sci. Toulouse, Math. 18 (2009), no. 4, p. 651-661.

[6] — - "Coleff-Herrera currents, duality, and Noetherian operators", Bull. Soc. Math. Fr. 139 (2011), no. 4, p. 535-554.

[7] M. Andersson \& R. LÄrkÄNG, "The $\bar{\partial}$-equation on a non-reduced analytic space", Math. Ann. 374 (2019), no. 1-2, p. 553-599.

[8] M. Andersson \& H. Samuelsson Kalm, "A Dolbeault-Grothendieck lemma on complex spaces via Koppelman formulas", Invent. Math. 190 (2012), no. 2, p. 261297.

[9] M. Andersson \& E. Wulcan, "Residue currents with prescribed annihilator ideals", Ann. Sci. Éc. Norm. Supér. 40 (2007), no. 6, p. 985-1007.

[10] - "Decomposition of residue currents", J. Reine Angew. Math. 638 (2010), p. 103-118.

[11] - "Direct images of semi-meromorphic currents", Ann. Inst. Fourier 68 (2018), no. 2, p. 875-900.

[12] D. BARlet, "Le faisceau $\omega_{X}$ sur un espace analytique $X$ de dimension pure", in Fonctions de plusieurs variables complexes, III (Sém. François Norguet, 1975-1977), Lecture Notes in Mathematics, vol. 670, Springer, 1978, p. 187-204. 
[13] J.-E. BJöRK, Analytic $\mathscr{D}$-modules and applications, Mathematics and its Applications, vol. 247, Kluwer Academic Publishers Group, Dordrecht, 1993, xiv+581 pages.

[14] - "Residues and $\mathscr{D}$-modules", in The legacy of Niels Henrik Abel, Springer, 2004, p. 605-651.

[15] N. R. Coleff \& M. E. Herrera, Les courants résiduels associés à une forme méromorphe, Lecture Notes in Mathematics, vol. 633, Springer, 1978, $\mathrm{x}+211$ pages.

[16] J.-P. Demailly \& M. Passare, "Courants résiduels et classe fondamentale", Bull. Sci. Math. 119 (1995), no. 1, p. 85-94.

[17] C. Dickenstein, Alicia; Sessa, "Canonical representatives in moderate cohomology", Invent. Math. 80 (1985), no. 3, p. 417-434.

[18] — , "Résidus de formes méromorphes et cohomologie modérée", in Géométrie complexe (Paris, 1992), Actualités Sci. Indust., vol. 1438, Hermann, 1996, p. 35-59.

[19] M. E. Herrera \& D. Lieberman, "Residues and principal values on complex spaces", Math. Ann. 194 (1971), p. 259-294.

[20] L. Hörmander, The analysis of linear partial differential operators. I, second ed., Grundlehren der Mathematischen Wissenschaften, vol. 256, Springer, 1990, Distribution theory and Fourier analysis, xii+440 pages.

[21] R. LÄRKÄNG \& H. SAmuelsson Kalm, "Various approaches to products of residue currents", J. Funct. Anal. 264 (2013), no. 1, p. 118-138.

[22] R. LÄRKÄNG \& E. WulCAN, "Residue currents and fundamental cycles", Indiana Univ. Math. J. 67 (2018), no. 3, p. 1085-1114.

[23] J. Ruppenthal, H. Samuelsson Kalm \& E. Wulcan, "Explicit Serre duality on complex spaces", Adv. Math. 305 (2017), p. 1320-1355.

[24] H. SAMUELSSON KALM, "The $\bar{\partial}$-equation, duality, and holomorphic forms on a reduced complex space", J. Geom. Anal. 31 (2021), no. 2, p. 1786-1820. 